\title{
Cintigrafía Esofágica y Pulmonar en Niños con Reflujo Gastroesofágico y Enfermedad Broncopulmonar
}

\author{
Drid. Maureen Rossel G.1-2; Dr. Sergio Ceresa 0.2; \\ Dra. Pilar Orellana B. 3; Dra. Cristina Pino 0.2. Dr. Patricio González. E., 3. \\ Dr. Enrique Olea G. ${ }^{3}$; Dra, Magdalena Araya Q.2-4; Dr. Julio Espinoza M. 2-4 \\ Esophageal and Lung Scintigraphy in Children with suspected \\ Gastroesophageal Reflux
}

\begin{abstract}
Thirty children (aged 2 months to 3 yedrs) with clinical evidence of gastroesophageal reflux (GER) and respiratory disease were investigatcd. Eighteen patients had normal nutritional status, four had mild undernutrition and eight were severely undernourished. Barium swallow and radionutide gastroesophageal and lung scintigrams were carried out in all patient. Barium swallow showed evidence of GliR in $86.6 \%$ esophageal seintigraphy in $90 \%$ and, the combined results of both methods in $100 \%$ of our cases. Pulmonary aspirates from esophageal contents were detected in $26.7 \%$ of the patients, all showing severe clinical and radiological evidence of pulmonary discasc. Our results suggest that radionuclide gastroesophageal and Jung stintigraphy are useful tools for the diagrosis of GFR and pulmonary aspiration, respcctively. However radiological studies remain necessary for the accurate description of the anatomical features of the csoplagus.
\end{abstract}

(Key words: GastroesophageaI reflux. Scintigraphy. Esophagus. Lung. Respiratory discasc).

El reflujo gastroesofágico (RGE) es frecuente en el niño menor 1-3. Algunos consultan por síntomas broncopulmonares de intensidad $y$ frecuencia variable en diferentes series ${ }^{4-5}$. En un estudio anterior mostramos que $26,7 \%$ de los pacientes que consultaban en Policlinico de Gastroenterología por RGE, tenian algún sintoma broncopulmonar y de ellos $2,2 \%$ sufrian crisis de cianosis 6

La relación entre RGE y aspiración pulmonar no ha sido debidanente evaluada en nuestro medio debido a la falta de técnicas precisas, sensibles y sencillas. Los exámenes con mayor rendimiento son el lavado brocoalveolar para buscar macrófagos cargados con lipidos 2 y la cintigrafía esofágica y pulmonar ${ }^{7-10}$. Esta última tiene algunas ventajas pues no es invasiva; permite observaciones más largas que la radiología con poca irradjación; no requiere sedación ni preparación especial del paciente; la administración del

1 Universidad de Chile, Departanento de Pediatría, División Ciencias Médicas Sur (Central).

2 Servicio de Pediatría, Hospital Paula Jaraquemada, Unidad Broncopulmonar y Gastroenterología.

3 Centro de Medicina Nuclear, Hospital José Joaquín Aguirre.

4 Universidad de Chilc, Instituro de Nutrición y Tecnología de los Alimentos. radiofármaco Tecnecio $99 \mathrm{~m}(\mathrm{~T} c 99 \mathrm{~m})$ se realiza en condiciones fissiológicas; produce resultados cuantificables y permite detectar aspiraciones pulmonares en volúmenes de $0.025 \mathrm{ml}$.

El objetivo de estc estudio fuc correlacionar los sintomas y signos clínicos con los hallazgos radiológicos y cintigráficos en pacientes en quienes se sospechaba RGE con aspiración pulmonar.

\section{PACIENTES Y METODOS}

Sc estudiaron 30 niños consecutivos de 2 meses a 3 años de edad. Todos ellos habían consultadio por vómitos, regurgitaciones, disfagia, tos noctuma o hipo (uno o varios de ellos) asociado con crisis de apnea, crisis de cianosis, episodios de obstrucción bronquial recidivante o neumonitis (uno o más de ellos).

Todos los pacientes fueron explorados mediantc estudios radiológicos de esófago, estómago y duodero (TEED) y cintigrafía del esótago y los pulmones. Esta última se realizó administrando $50 \mathrm{Ci} / \mathrm{Kg}$ (mínimo $500 \mathrm{Ci}$ ), de $\mathrm{Te} 99 \mathrm{~m}$ sulfuro coloide en la mitad del volumen habitual de mamadera de cada niño, reservando el resto sin Tc $99 \mathrm{~m}$ para su administración posterior de modo de limpiar faringe y esófago de material radioactivo. Las imágenes se registraron durante una hora con frecuencia de 360 imágenes de 10 segundos $\mathrm{c} / \mathrm{u}$ por hora ${ }^{8-11}$ (Fig. 1). 


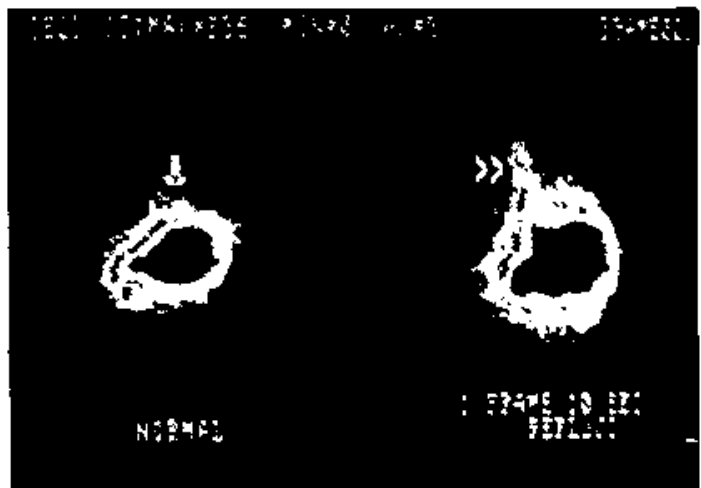

Figura I.

Indgencs computarizadas para mostrar at aspectos de un estónago normal ( t) y la aparición de reflujo. (*)

El procesamiento de los datos en la computadora incluyó la confección de curvas tiempo actividad para regiones de inte rés dibujadas sobre el esófago u otra área que se deseara estudiar en profundidad. Para el análisis de estas curvas se aceptó como positivo para RGE un alza sobre el $100 \%$ de activiđad en relación a una basal. La basal corresponde a las 3 imagenes obtenidas inmediatamente antes de producirse el episodio de RGE (lig. 2).

Para el estudio de aspiración pulmonar se tomaron imágenes 2 horas después de la administración del radiofármaco. En la última alimentación de esa noche se reforzó la dosis de tecnecio, administrando coloide suficiente para llegar hasta $2 \mathrm{mCl}$. Dieciocho horas después de la primera dosis se tomaron nuevas imágenes en proyecciones antcroposterior $y$ posteroanterior del torax. con un tiempo de adquisición de datos de 5 minutos (Fig. 3).

\section{RESULTADOS}

De los 30 niños estudiados 18 eran eutróficos, 4 desnutridos Grado 1 y 8 desnutridos Grado II (según tablas del National Center of Health Statistics j1 ${ }^{3}$.

Sólo en dos de los pacientes estudiados no se encontraron síntomas digestivos, uno tenía disfagia $\mathrm{y} \operatorname{los} 2$ ? restantes vómitos, regurgitaciones o ambos. Habian síntomas de obstrucción bronquial recidivante (SBOR) en 18 pacjentes ( $60 \%)$, evidencia de neumonitis en $3(10 \%)$, antecedentes de crisis de asfixia en $7(23,3 \%)$ y neumonía en $2(6,7 \%)$. EI TEED demostró RGE en 26 $(86,6 \%)$ de los niños estudiados y la cintigrafía dio resultados positivos en 27 (90\%), incluyendo
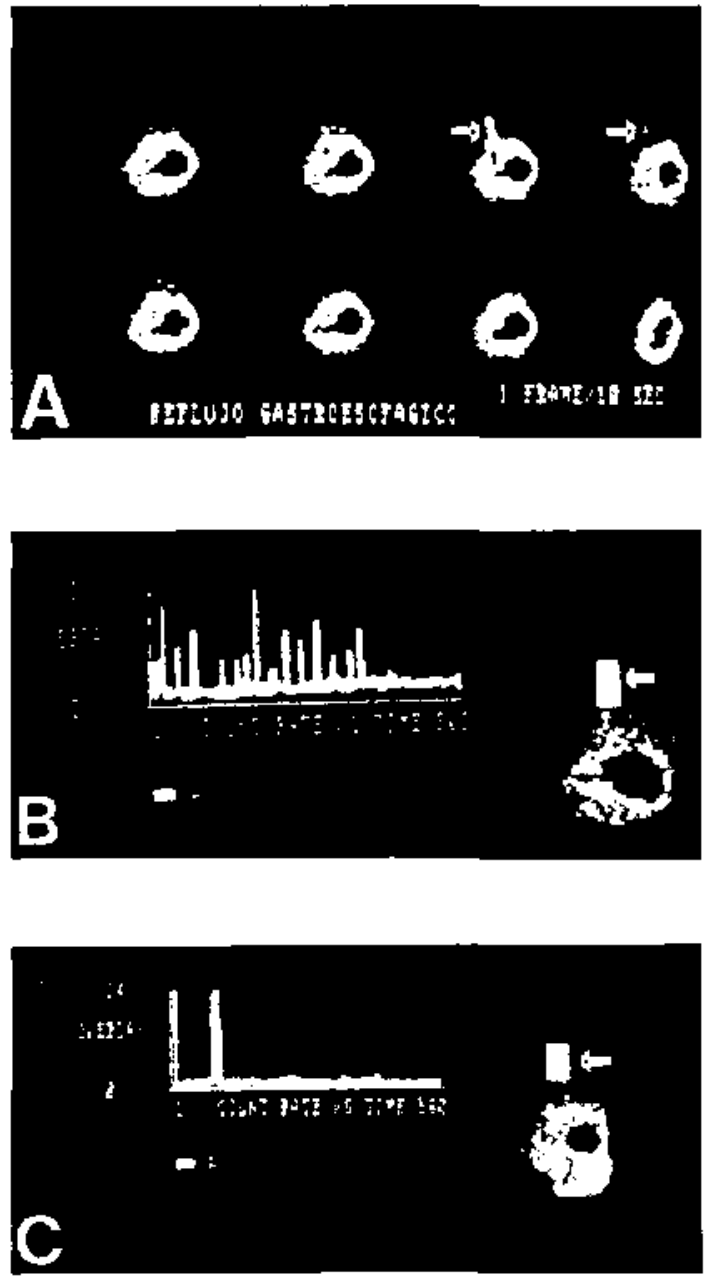

Fìgura 2 .

A. Imágenes computarizadas de 10 segundos de duración cadd und. La flecha blanca musstra la aparición de RGE B. Selecciona un árca de interés $(\uparrow)$ a la que se le realiza una curva ticmpo-actividad durante 1 hora (360 inágenes). Se obscrvan numierosos episodios de RGJi sobre la linea de activid ad basal.

C. Curva de tiempo-actividad efectuada en otro paciente. en que se observa sólo un episodio de RGF.

los cuat ro sin evidencia de reflujo en el TEED. A la inversa los tres pacientes con cincigrafía negativa tenian reflujo en la serie radiográfica. La cintigrafía pulmonar mostró aspiración de mate. rial radioactivo en 8 (26,7\%) niños (Fig. 3): en todos ellos los sintomas clínjcos eran más severos y prolongados; siete tenían simultáneamente evidencia de neumonitis, atelectasias o ambas y uno bronquitis deformante; cinco de estos ocho eran desnutridos. 


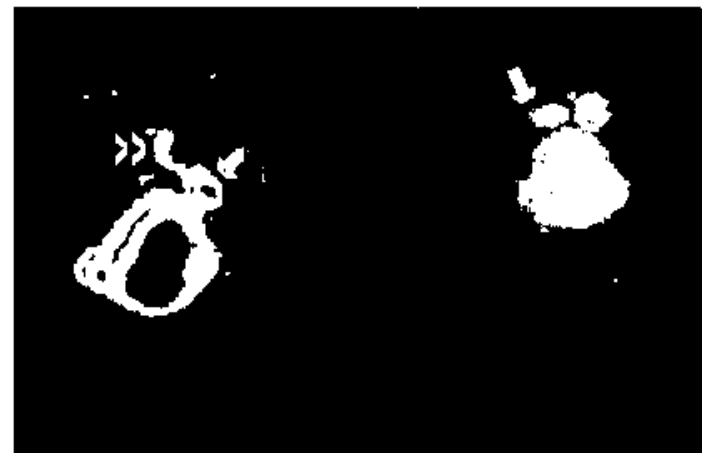

Figura 3.

Ilustra las imágenes de aspiración pulmonar presentadas a las 2 y 18 horas en uno de los pacientes.

a) A las dos horas de observación se visualiza una imagen de aspiración en la base pultiona izquierd a $(\uparrow)$ y dibujo de la vía aérea superior ( $)$.

b) A las 18 horas aparece una segunda inagen de aspiración ( $\uparrow$ ) en la basc pulmonar derecha.

\section{COMENT ARIO}

La cintigrafia esofágica y pulmonar con $\mathrm{T}_{\mathrm{c}}$ $99 \mathrm{~m}$ sulfuro coloide es un métado no invasivo, de baja irradiación, bien tolerado por el enfermo y adecuado para evaluar el RGE y aspiración pulmonar en el nifĩo. Rudd, Christic 10 y Hey. man9 describieron una sensibilidad de $80 \%$ y $59 \%$ respectivamente. Al combinar la cintigrafía csofágica con el TEED, ambos autorcs obtuvieron resultados positivos en 88 a $90 \%$ de los casos, resultando semejante al de la prueba de Reflujo ácido de Tuttle ${ }^{1-7}$ y la medición continua del $\mathrm{pH}$ en 24 horas ${ }^{\text {4-15 }}$. En nuestro estudio la sensibilidad de ambos métodos fuc mayor: $86,6 \%$ para la radiografía y $90 \%$ para la cintigrafía, y al combinar ambos métodos, el $100 \%$ de los enfermos tenía alguno de ellos positivo.

La cintigrafía esolágica para detectar $\mathrm{RGE}$ tiene sensibilidad semejante al TEED, en nuestra serie. Sin embargo, el TEED es importante $y$ hasta ahora insustituible, pues muestra aspectos anatómicos no detectables con la cintigrafía. A su vez, la cintigrafía esofágica permite periodos de observación prolongados y continuos (1 hora), lo que define tnejor el número de episodios e intensidad del RGE para un determinado período de tiempo. Así, es posible medir con más precisión la severidad del reflujo, como lo hace la medición continua del pH en 24 horas. Con esta técnica es también posible evaluar la respuesta terapéutica, mediante estudios serjados.

La aspiración pulmonar fue detectada mediante la técnica de cintigrafía pulmonar en 8 enfer- mos $(26,7 \%)$, coincidiendo con los casos de mayor severidad de las molestias pulmonares y el RGE. Esta experiencia coincide con la obtenida cn un grupo de pacientes semejantes ${ }^{16}$. Sin embargo en ésa se encontró un enfermo cuyas cintigrafías, esofágica y pulmonar, eran positivas con TEED negativo, situación que no se dio en nuestra seric y que implica una ventaja adicional de la combinación de cintigrafía esofágica y pulmonar.

Un resultado de cintigraf ia negativo para aspiración pero positivo para RGE, en un nirio con enfermedad bronoopulmonar severa, sugiere la necesidad de orientar el estudio del trastorno broncopulmonar hacia otras posibilidades etiológicas, sin perjuicio de repetir la cintigrafia.

La sensibilidad del TEED para detectar RGE es variable1:9.10. En diferentes series oscila entre 38 y $85 \%$ de los niños con síntomas sugerentes. En nuestra serie alcanzó el $86,6 \%$ probablemente debido al uso de técnias estándar, a la larga experiencia del radiólogo con el trastorno y al selccto grupo de enfermos que constituyen la muestra. Es importante destacar que en 10\% de los niños el RGE se demostró con TEED y no por cintigrafía esof́́gica y que en $13,3 \%$ ocurrió la situación contraria.

En conclusión, la cintigrafía gastroesofágica es sensible para detectar la presencia de RGE y permite diagnosticar la aspiración pulmonar secundaria, si se combina simultáneamente con cintigrafía pulmonar. El estudio no es invasivo, se realiza en condiciones físiologicas y con baja irradiación.

\section{RESUMEN}

Se evaluaron 30 niños ( $2 \mathrm{~m}$. a 3 años de edad) con RGE clínico y sintomas broncopulmonares severos, 18 eutróficos, 4 y 8 desnutridos grado 1 y 2 respectivamente. A todos se les hizo TEED y Cintigrafía esofägica y pulmonar con Tc $99 \mathrm{~m}$. El TEED fue positivo para RGE en $86,6 \%$ de los pacientes y la cintigrafia esofágica en el $90 \%$ de ellos. La combinación de ambos métodos alcanzó el $100 \%$ de positividad. La aspiracjón pulmonar contemporánea fue positiva en $26,7 \%$ de los enfermos. Todos habían presentado curso clinico broncopulmonar severo, que en 8 de ellos había sido confirmado radiológicamente. Concluimos que la cintigrafía esofágica y pulmonar es una nueva $c$ importante herramienta en el diagnóstico de RGE y aspiración pulmonar respectivamente. Sin embargo, sugerimos combinar. lo con estudio radiológico para definir mejor la anatomia, mientras se acumula mayor experien. cia con la técnica cintigráfica. 


\section{REFERENCIAS}

1 Herbst, J.J.: "Gas1roesophageal Reflux". J. Pediatr. 98: 859, 1981.

2 Herbst, J.J.: "Reflujo gastroesofágico y enfermedad pulmonar" Pediatrics (ed. esp.) 12:5, 1981.

3 Berquist, W., Rachelefsky, G., Kadden, M., Siegel, $S$ Katz, R. Fonkalsrud, E, Ament, M.: "Reflujo gastroesofágico asociado a Neumonía recidivante y asma crónica en los niños". Pediatrics (ed, esp.) 12 : $38,1981$.

4 Liler, A.R., Byme W.J., Ament M.E.: "Recurren1 pulmonary disease in children: a complication of gastroesophaged reflux". Pediatrics 63: 47, 1979.

5 Donus O., Casar C., Larraín A.: "Esophageal reflux an unrecognized cause of recurrent obstructive bronchitis in children". J. Pediatr. 89: 220, 1976.

${ }^{6}$ Rossel, M., Cereza, S., Espinoza, J.: Reflujo gastroesofágico: Evolución clínica y radiológica. Rev. Chil. Pediatr. 54: 16, 1983

7 Arasu T.; Wyltie R., Fitzgerald $J$, Franken $E$., Sideliqui L., Lehman $G$., Ligen H., Grosfeld J.: "Gastroesophageal reflux in infants and children comparative accuracy of diagnostic methods". J. Pediatr. 96: 798, 1980.

8 Fisher R.S., Malmud L.S., Roberts G.S., Lobis I.F.: "Gastroesophageal scintiscanning to detect and quantitate G.E. reflux Gastroenterology 70: 301, 1976 .
9 Heymon S., Kirkpatrick J., Winter H., Treeves S. "An improved radionuclide method for the diagnosis of gastroesophageal sefux and aspiration in children (Milk Scan). Radiology 13]: 479, 1979.

10 Rudd, $T$, Christie, D.: "Demonstration of gastroeso. phageal reflux by radionuclide gastroesophagography". Radiology 131: 483, 1979.

11 Sty, J., Starshok, R.: "The Role of Radionuclide studies in Pediatric Gastrointestinal disorders". Seminars in Nuclear Medicine Vol. XII No 2 (April), 156, 1982.

12 Orellana P., Olea, E, Ceresa, S., Rossel, M., Pino, C; González, P.; Araya, M., Espinozo, J., Otárola, $S$ : Evaluación de la técrica radioisotópica en el estudio del tracto digestivo alto. XIV Congreso Chileno de Pediatria. Vina del Mar. 1983:

13 U.S. Department of Health N.C.H.S. Growth charts Monthly Vital Statistics Report. Vol. 25, No3. 1976.

14 Ramerofsky, R.L., Leape, L.L.: Continuons upper esophageal $\mathrm{pH}$ momtoring in infants and children with gastroesophageal reflux, penumonia and apneic spells. J. Pediatr. Suxg. 16: 374, 1981.

15 Siebert, J.J., Burne, W.J., Liler, A.R., Lathre, T, Leach, M. Compbell, M.: "Gastroesphageal Reflux The Acid Test: Scintigraphy or the pH probe? A.J.R. 140: 1087, 1983.

16 Boayaprapa S., Alderson Ph., Garfinkel D., Chipps $B$, Wogner $H .:$ "Detection of Pulmonary Aspiration in infants a children with Respiratory Disease: Concise communication". J. Nucl. Med. 21: 314-318, 1980 . 